\title{
The Effect of Interruptions and Breaks on Insight and Impasses: Do You Need a Break Right Now?
}

\author{
Flora Beeftink and Wendelien van Eerde \\ Eindhoven University of Technology \\ Christel G. Rutte \\ Tilburg University
}

\begin{abstract}
Some time away from a problem, or incubation time, is found to be beneficial to creative problem solving. But are interruptions as equally helpful as breaks? An experiment was conducted to gain more insight into the differences between imposed and selfinitiated breaks, and their effects on creativity, specifically on impasses and insights. There were three experimental conditions, (a) a continuous condition, in which participants were not allowed to switch back and forth between tasks, (b) an interruption condition, in which participants had to switch tasks at a predetermined moment, and (c) a break condition, in which participants could switch tasks at their own discretion. Results showed that taking breaks at moments chosen at one's own discretion led to solving more insight problems and reaching fewer impasses than at moments that were chosen by others. Furthermore, compared to working continuously, interruptions led to fewer impasses, but not to solving more insight problems.
\end{abstract}

At work, most knowledge workers can switch between different activities or take breaks at their own discretion, and they are also regularly subjected to interruptions initiated by others (Czerwinski, Horvitz, \& Wilhite, 2004; Nandhakumar \& Jones, 2001). Jett and George (2003) theorized that self-initiated breaks from work can have positive effects on creativity, whereas interruptions initiated by others may not bring about these positive effects. This proposition has not yet been tested. This article describes an experiment to gain more insight into the differences between imposed and self-initiated breaks, and their effects on creativity, specifically on insights (i.e., the distinctive and apparently sudden realization of a strategy that aids in solving a problem; Sternberg \& Davidson, 1999), and on impasses (i.e.,

Wendelien van Eerde is currently at the Amsterdam Business School, University of Amsterdam.

Correspondence should be addressed to Flora Beeftink, Department of Technology Management, Subdepartment of Human Performance Management, Eindhoven University of Technology, P.O. Box 513, 5600 MB, Eindhoven, The Netherlands. E-mail: f.beeftink@tue.nl the feeling of being "stuck" and not being able to solve a problem; Fleck \& Weisberg, 2004, p. 998).

\section{INSIGHT PROBLEM SOLVING}

Having a sudden insight, or "eureka moment," is a wellknown phenomenon in everyday life and in the literature (Sternberg \& Davidson, 1999). Individuals suddenly and unexpectedly get a good idea that brings them a great step further in solving a problem (Wallas, 1926). To be more specific: Individuals may suddenly become aware of a new strategy that may solve the problem at hand (Sternberg \& Davidson, 1999). An often cited creative problem-solving model that includes sudden insights is that of Wallas (1926). It consists of four stages: (a) the preparation stage: gain in-depth knowledge about a problem, (b) the incubation stage: take some time away from the problem, (c) the illumination stage: suddenly and unexpectedly an insight comes to mind, and (d) the verification stage: verify the applicability of the idea. The underlying hypothesis of this model is that incubation time aids the problem-solving 
process. In a review article about experimental research on the effect of incubation time, Dodds, Ward, and Smith (in press) concluded that this incubation effect exists: roughly $75 \%$ of the experiments show positive effects of incubation time.

In addition to Wallas' model, Smith and Blankenship (1991) and Smith (1995) suggested that incubation time is only valuable in specific situations: "Problems that are solved immediately require no incubation [time]" (Smith \& Blankenship, 1991, p. 63). They argued that individuals try to tackle problems with a certain problem solving strategy, and that it is very difficult for them to approach a problem with a different strategy when the first one does not seem to be appropriate. They refer to this block to successful problem-solving as a cognitive state of fixation. In four experiments, Smith and Blankenship showed that incubation time was only helpful in situations in which individuals needed to overcome this state of fixation.

The insight problem solving model shown in Figure 1 combines Wallas's model (1926) with the ideas of Smith and Blankenship (1991) and Smith (1995). First, individuals start working on a problem, which results in some problem progress. This is similar to what Wallas called the preparation stage. If the problem is tackled with the appropriate problem solving strategy, it can be solved at once. If not, individuals reach a cognitive state of fixation. They can keep trying to solve the problem, but the chance that they will find a suitable strategy to tackle the problem is very small and, most likely, the problem will remain unsolved. Another option is to take some incubation time. A sudden insight might occur that reveals a new strategy which may solve the problem.

\section{The Effect of Interruptions and Breaks on Insights}

Both interruptions and breaks give individuals the opportunity to take some time away from a problem, in other words, to have some incubation time. As previous research has suggested (Dodds et al., in press), either form of incubation time should improve problem solving performance. Therefore, the first hypothesis of this article states that individuals who are interrupted benefit from the incubation effect, and solve more problems than individuals who continuously work on

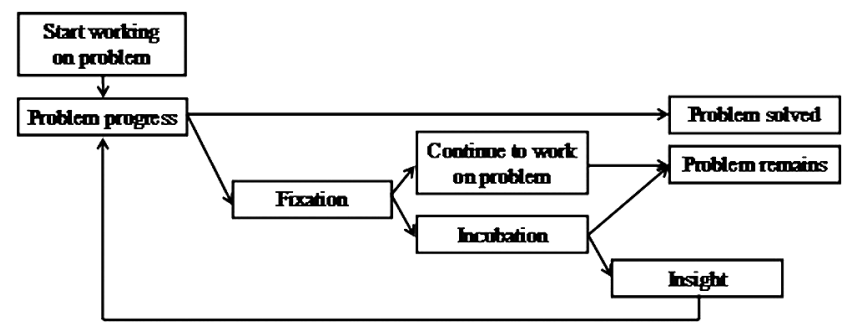

FIGURE 1 Insight problem solving model. these problems. Similarly, a second hypothesis is that individuals who can take breaks at their own discretion solve more problems than individuals who continuously work on these problems.

Hypothesis 1: Individuals who are interrupted solve more insight problems than those who work continuously on them.

Hypothesis 2: Individuals who can take a break at their own discretion solve more insight problems than those who work continuously on them.

Not empirically tested before is the question whether there is a difference between the effects of interruptions and breaks on creative performance, specifically, whether breaks should be preferred over interruptions. Interruptions are generally seen as negative and disruptive, whereas breaks are not (Jett \& George, 2003). In line with this belief, time management training advises to avoid interruptions as much as possible (Green \& Skinner, 2005; Macan, 1994). Some research confirms the proposed negative effects on task performance. For example, in a field study in a commercial telecommunications office, Eyrolle and Cellier (2000) found that operators needed more time to complete tasks that were interrupted, compared to tasks that could be carried out without interruptions. They also conducted an experiment that revealed that interruptions also have a negative effect on the number of errors individuals make. Recently, Bailey and Konstan (2006) have confirmed both of these findings in a similar experiment. They found that interrupted individuals needed more time to complete their tasks, compared to uninterrupted individuals, and that they made twice as much errors. But not all research finds negative effects of interruptions on task performance. In a field study among 46 bank managers, König, Kleinmann, and Höhmann (2004) found no relation between daily performance and interruptions. Zijlstra, Roe, Leonora, and Krediet (1999) conducted two experiments in which they found that interruptions caused individuals to work faster while maintaining the same level of quality.

In conclusion, former research suggests that, opposed to the positive effect of incubation, interruptions might also cause negative effects on task performance. Self-initiated breaks, on the other hand, may not bring about such negative effects because individuals can choose a convenient point in time to take them. Therefore, a third hypothesis proposes that individuals who can take a break at their own discretion outperform individuals who are interrupted.

Hypothesis 3: Individuals who can take a break at their own discretion solve more insight problems than those who are interrupted. 


\section{THE FEELING OF BEING STUCK}

Conceptually new to this field of research is the distinction between the cognitive state of fixation and the psychological state of impasse, and the use of impasses as a dependent variable in creativity research. An impasse is defined as the feeling of being "stuck" (Fleck $\&$ Weisberg, 2004), and can be differentiated from the cognitive state of fixation in two ways: First, the psychological state of impasse is affective and incorporates negative emotions such as confusion and frustration (Fleck \& Weisberg, 2004), whereas the cognitive state of fixation is free of such emotions because it occurs unconsciously. Second, a lagged effect might exist between the moment of fixation and the perception of reaching an impasse: After fixation, individuals might not realize immediately that it will be difficult to solve the problem. Instead, it might take some time before they feel stuck. In an experiment by Segal (2004), for example, participants worked up to twenty minutes on the presented insight problem before they stated that they had reached an impasse. To be more precise, we suggest that, after individuals get into a state of fixation, negative emotions gradually increase over time. When confusion and frustration reach a certain level, individuals decide that they do not want to work on the problem anymore and give up. At this point in time, they reach the psychological state of impasse.

In previous research on the incubation effect, both the cognitive state of fixation and the psychological state of impasse were predominantly treated as a necessary precondition for the incubation effect to occur (Dodds et al., in press). In this article, the two are conceptually separated: Fixation is treated as a necessary precondition, but impasse as an affective outcome of the creative process, worthwhile to be studied in more detail.

\section{The Effect of Interruptions and Breaks on Impasses}

Both interruptions and breaks might be of influence on the process of reaching an impasse. After individuals reach the cognitive state of fixation, interruptions or breaks may bring the process of building up negative emotions to a temporary halt. This halt might prevent these emotions from reaching the critical level of impasse. Assuming that individuals reach a state of fixation if they are not able to solve an insight problem right away (Smith, 1995; Smith \& Blankenship, 1991), Hypothesis 4 states that individuals who are interrupted report impasses less often than individuals who continuously work on problems. Similarly, a fifth hypothesis is that individuals who can take a break at their own discretion report impasses less often than individuals working continuously.
Hypothesis 4: Individuals who are interrupted report fewer impasses than those who work continuously on an insight problem.

Hypothesis 5: Individuals who can take a break at their own discretion report fewer impasses than those who work continuously on an insight problem.

The last hypothesis focuses on the question of whether there is a difference between the effects of interruptions and breaks on impasses reached, and which is to be preferred in order to prevent an impasse. Here the emphasis lays on the affective influences of interruptions and breaks, and whether these add to the negative feelings leading to the impasse. Breaks are not expected to be a source of negative emotions, but interruptions may be. For example, in the experiment of Bailey and Konstan (2006), interrupted participants felt more annoyed and anxious than uninterrupted participants. Similarly, Zijlstra et al. (1999) found that interruptions had a negative effect on their participants' emotions. On the other hand, interruptions may also prevent feelings of boredom when individuals are working on simple tasks (Fisher, 1998). To summarize, it is expected that, although interruptions may reduce feelings of frustration, they may also be a source of negative emotions. Therefore, the sixth hypothesis states that individuals who can take a break at their own discretion feel fewer negative emotions than individuals who are interrupted, and, as a consequence, they reach the state of impasse less often.

Hypothesis 6: Individuals who can take a break at their own discretion report fewer impasses than those who are interrupted.

\section{METHOD}

\section{Participants}

One hundred and nine Dutch undergraduate students (12 female) participated in the experiment. The average age of the participants was 20.8; the youngest was 19, and the oldest 27 years old. Their major was in industrial engineering and management science and they were highly educated in math and statistics.

\section{Task}

The creative task for each of the students was to solve three insight problems. Different types of insight problems exist: verbal, mathematical, and spatial problems (Dow \& Mayer, 2004). We decided not to use mathematical or spatial insight problems, such as the well known nine dot problem (Runco \& Sakamoto, 1999; 
Sternberg \& Davidson, 1999), because of the risk that these would be solved mathematically instead of through insight (for comments on hybrid insight problems, see Weisberg, 1995). This risk was particularly large in our student sample. Therefore, verbal insight problems were used: three relatively difficult cryptic crossword clues. ${ }^{1}$ An example is: "not seeing the window covering (5 characters)" is "blind." The three word puzzles were selected and pretested during a pilot study among 36 undergraduate students with the same educational background. Pretesting showed answer patterns typical for insight problems: Solutions were generally found right away, in the first minute $(55 \%)$, or when participants came back to a word puzzle after working on other word puzzles $(21 \%)$.

\section{Design}

The design consisted of three conditions. Participants in all conditions had a total of three times six minutes to work on the word puzzles. Participants in the continuous condition were not allowed to switch back and forth between the word puzzles, which meant that they worked six minutes on puzzle $\mathrm{A}$, then six minutes on puzzle $\mathrm{B}$, and then six minutes on puzzle $\mathrm{C}$ (see Figure 2). In the interrupted condition, participants had to switch to the next puzzle after three minutes, and came back to it after they had worked on the other two puzzles for three minutes each. The sequential ordering of the puzzles was A, B, C, A, B, C (see also Figure 2). Note that both the participants in the continuous and the interrupted conditions worked continuously on a puzzle during the first three minutes. Differences between these conditions occurred only after the third minute. In the break condition, participants could switch back and forth between the puzzles at their own discretion. An example of a possible sequence of puzzles in this last condition is also shown in Figure 2.

\section{Procedure}

Each participant was accompanied by an observer. Beforehand, the observers handed out and verbally repeated written instructions regarding the goal and procedure of the assignment. The participants were instructed to notify their observer when a puzzle was solved, or when they had reached an impasse. Participants were debriefed at the end of the experiment

\footnotetext{
${ }^{1}$ In cryptic crosswords, each individual clue is a word puzzle in and of itself. In this case, the clues are the insight problems, not to be mistaken with clues or hints that are sometimes used as intervention to aid insight problem solving (e.g., Chronicle, Ormerod, \& MacGregor, 2001).
}

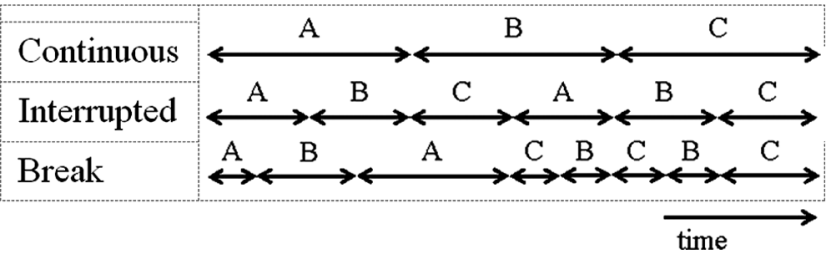

FIGURE 2 Experimental design, task sequences.

and the solutions to the puzzles were sent to them by e-mail the next day.

\section{Measures}

The observers recorded solutions and impasses. Because the continuous condition and the interrupted condition differed only after the third minute of a puzzle's allotted time, they distinguished between solutions and impasses that occurred during the first half (first $3 \mathrm{~min}$ ) and the second half (last $3 \mathrm{~min}$ ) of a puzzle's allotted time. As is customary when an experimental task consists of multiple insight problems (Dodds et al., in press), the data of the three puzzles were combined prior to dataanalysis: For each participant, we added the number of puzzles that had been solved during the first half of their allotted time. Similarly, the number of solutions per participant during the second half was calculated. The same procedure was used to calculate the number of impasses during the first and second halves of the puzzles' allotted time.

\section{RESULTS}

\section{Solutions}

In total, 85 word puzzles were solved, which means that, on average, participants solved less than one out of the three puzzles. Sixty-three puzzles were solved during the first three minutes that had been spent on them, and 22 during the last three minutes. Specifically, half of the puzzles $(n=44)$ were solved right away, during the first minute, confirming that these were insight problems: Either a solution was found immediately, or it was unlikely that it would be found at all. Means and standard deviations of the number of solutions per participant for each of the three conditions during the first three minutes (first half) and the last three minutes (second half) are presented in Table 1. Significant differences between the conditions are marked with different superscripts.

Although most puzzles were solved during the first half of their allotted time, no differences were expected between the three conditions during this first half. A one-way ANOVA confirmed this: No significant differences were found between the groups, $F(2,108)=.47$, 
TABLE 1

Means and Standard Deviations of Solutions and Impasses

\begin{tabular}{|c|c|c|c|c|c|c|c|c|c|}
\hline & & \multicolumn{4}{|c|}{ Solutions } & \multicolumn{4}{|c|}{ Impasses } \\
\hline & & \multicolumn{2}{|c|}{ 1st Half } & \multicolumn{2}{|c|}{ 2nd Half } & \multicolumn{2}{|c|}{ 1st Half } & \multicolumn{2}{|c|}{ 2nd Half } \\
\hline & & $M$ & $S D$ & $M$ & $S D$ & $M$ & $S D$ & $M$ & $S D$ \\
\hline Continuous & $(N=36)$ & $.62^{a}$ & .69 & $.08^{a}$ & .28 & $.31^{a}$ & .88 & $.94^{a}$ & .95 \\
\hline Interrupted & $(N=36)$ & $.67^{a}$ & .80 & $.14^{a}$ & .35 & $.26^{a}$ & .54 & $.39^{b}$ & .69 \\
\hline Break & $(N=36)$ & $.51^{a}$ & .63 & $.39^{b}$ & .64 & $.36^{a}$ & .63 & $.28^{c}$ & .45 \\
\hline
\end{tabular}

Note. Different superscripts indicate significant differences between conditions, $p<.05$ (one-tailed).

$p=.63$. A second one-way ANOVA revealed differences between the groups during the second half of the allotted time, $F(2,108)=4.63, p=.01, \omega=.30$.

Scheffe's tests were conducted to evaluate pairwise differences among the conditions during this second half. Participants in the continuous condition did not solve more puzzles than participants in the interrupted condition, $p=.87, n s$. This means that Hypothesis 1 was not confirmed; interruptions were not related to more problems solved.

In the break condition, all but one participant took breaks from the puzzles they were working on: They started to work on a puzzle, worked on one or more other puzzles temporarily, and then got back to the first one. On average, they switched between puzzles nine times. During the second half of the allotted time, participants in the break condition solved more puzzles than participants in the continuous condition, $p=.010$ (onetailed). Hypothesis 2 was confirmed, which means that positive effects of breaks on problem solving performance were found. Furthermore, participants in the break condition solved more problems than participants in the interrupted condition, $p=.035$ (one-tailed). Hypothesis 3 was also confirmed: Individuals who could take a selfinitiated break outperformed the interrupted individuals.

\section{Impasses}

Ninety-two impasses were reported, which means that, on average, almost all participants reached an impasse. ${ }^{2}$ Thirty-four impasses were reported during the first half of the allotted time and 58 during the second half. The fact that more participants felt stuck during the second half supports our assumption that it takes some time to reach the psychological state of impasse. Means and standard deviations of the number of reported impasses per participant for each of the three conditions during the first and the second half of the allotted time are presented in Table 1.

\footnotetext{
${ }^{2}$ One outlier was excluded, because this participant reported nine impasses.
}

Again, effects were only expected during the second half of the allotted time. A one-way ANOVA showed no differences between the three conditions during the first half of the allotted time, $F(2,108)=.18, p=.84$, $n s$, and did reveal differences between the groups during the second half of the allotted time, $F(2,108)=8.66$, $p<.000, \omega=.42$.

Scheffe's tests were conducted to evaluate pairwise differences among the conditions during this second half. Participants in the interrupted condition reported fewer impasses compared to the participants in the continuous condition, $p=.004$ (one-tailed). This means that Hypothesis 4 was confirmed; interruptions reduced the number of reported impasses. The participants in the break condition also reported fewer impasses than the participants in the continuous condition, $p=.001$ (one-tailed). This means that Hypothesis 5 was confirmed; breaks also reduced the number of impasses. Furthermore, the participants in the break condition reached fewer impasses than the participants in the interrupted condition, $p=.035$ (one-tailed). Thus, Hypothesis 6 was also confirmed: Taking a break at one's own discretion had additional positive effects over being interrupted.

\section{DISCUSSION}

The purpose of this study was to gain more insight into the differences between imposed and self-initiated breaks, and their effects on creativity, specifically, on insight and impasses. The results reveal two major differences: Individuals who took breaks at their own discretion (a) solved more problems and (b) reached fewer impasses than interrupted individuals. It was also found that interrupted individuals reached fewer impasses than individuals who worked continuously on problems.

\section{Insight Problem Solving}

These results add to the literature on insight problem solving and incubation time. First, the findings confirm the existence of the incubation effect suggested by 
Wallas (1926), in line with earlier research on this topic (Dodds et al., in press; see also Segal, 2004). Second, they are a unique contribution, because they imply that the incubation effect is primarily evident when individuals can take incubation time at their own discretion, and not when others determine the moment at which the task is interrupted.

One possible explanation might be that a self-chosen moment to switch tasks allows individuals to end their cognitive activities on one task and start working on the next, whereas an interruption may not allow for such closure, and temporarily forces individuals to divide their cognitive resources (Norman \& Bobrow, 1975). This may have negative influences on task performance (Bailey \& Konstan, 2006; Eyrolle \& Cellier, 2000). If so, the beneficial effect of the incubation period on the creative problem solving process might be undone by the negative effect of being interrupted. A second possible explanation might be that the incubation effect is stronger when individuals intentionally choose to take some incubation time. In a survey among 213 college professors, Wells (1996) found that those who intentionally set manuscripts aside for some time produced more published articles than those who did not. More research is needed to replicate our results and investigate the precise mechanisms responsible.

The results have important implications for further research on the incubation effect. In previous research, interruptions, rather than self-initiated breaks, were used in experimental designs: Generally, participants were not allowed to choose when they switched to another task and back. Instead, they had to switch tasks at predefined moments (Dodds et al., in press). Exceptions are some early studies on incubation (e.g., Olton, 1979; Patrick, 1938) and a recent study by Christensen and Schunn (2005), in which participants were free to move back and forth between tasks. Other exceptions are experiments in which participants were allowed to switch tasks after they had reached an impasse (e.g., Butler \& Thomas, 1999; Segal, 2004; Seifert, Meyer, Davidson, Patalano, \& Yaniv, 1995). Considering our results, it might be preferable to incorporate breaks instead of interruptions in future experimental designs when studying the incubation effect.

\section{The Feeling of Being Stuck}

A second unique contribution of this article is that it is one of the first experimental studies in which impasses, or feelings of being stuck (Fleck \& Weisberg, 2004), are considered to be an affective outcome of the creative process, and have therefore been measured as a dependent variable. The results of this study suggest that both interruptions and breaks help to prevent individuals from reaching an impasse, and that individuals who take breaks at their own discretion reach even fewer impasses than interrupted individuals.

Presumably, the underlying processes of these positive effects are associated with emotions, rather than cognitions. If individuals use a faulty problem solving strategy, negative feelings, such as frustration and confusion, might build up gradually and result in an impasse when they reach a certain critical level. Both interruptions and breaks might prevent the negative emotions from reaching this critical level. Emotions may also explain why breaks are to be preferred over interruptions. The division of cognitive resources over the main task and the second, interrupting task (Norman \& Bobrow, 1975) can cause an increased level of stress (Kirmeyer, 1988) and negative emotions (e.g., Bailey \& Konstan, 2006; Zijlstra et al., 1999). When these are added to the negative emotions caused by the unsolvable problem, interrupted individuals might reach the critical level of impasse at an earlier moment than individuals who are able to take breaks at their own discretion.

In future research, the psychological state of impasse might be an interesting concept for further investigation. Specifically, it may be interesting to focus on both the psychological state of impasse and the cognitive state of fixation and see how these relate to each other, to performance measures, and to other psychological constructs. Fixation might be an important construct with regard to problem solving and creative performance, and negative psychological effects are to be expected from impasses. To gain more insight into the difference between fixation and impasse, one could conduct an experiment similar to that of Fleck and Weisberg (2004), in which participants were asked to think aloud while solving an insight problem. Fleck and Weisberg primarily focused on the processes leading to insight, but a future experiment could focus on faulty problem solving strategies (fixation) and the development of emotions leading to the feeling of being stuck.

Interruptions are generally considered to have an unfavorable effect on performance (Jett \& George, 2003). Therefore, time management training advises individuals to prevent them as much as possible (Green \& Skinner, 2005; Macan, 1994). Although the laboratory results of the current study may not be fully generalizable to creative tasks in a work setting, they imply that, in certain situations, interruptions may be beneficial. When individuals need to work on a problem on which they do not make much progress, an interruption may prevent high levels of frustration and a feeling of being stuck. Yet, we found that taking a break at one's own discretion is preferable to being interrupted. 


\section{REFERENCES}

Bailey, B. P., \& Konstan, J. A. (2006). On the need for attention-aware systems: Measuring effects of interruption on task performance, error rate, and affective state. Computers in Human Behavior, 22, 685-708.

Butler, D. L., \& Thomas, K. M. (1999). Preliminary study of the effectiveness of some heuristics used to generate solutions to illstructured problems. Psychological Reports, 84, 817-827.

Christensen, B. T., \& Schunn, C. D. (2005). Spontaneous access and analogical incubation effects. Creativity Research Journal, 17, 207-220.

Chronicle, E. P., Ormerod, T. C., \& MacGregor, J. N. (2001). When insight just won't come: The failure of visual cues in the nine-dot problem. Quarterly Journal of Experimental Psychology Section A: Human Experimental Psychology, 54, 903-919.

Czerwinski, M., Horvitz, E., \& Wilhite, S. (2004, April). A diary study of task switching and interruptions. Paper presented at the CHI, Vienna, Austria.

Dodds, R. A., Ward, T. B., \& Smith, K. G. (in press). A review of experimental research on incubation in problem solving and creativity. In M. A. Runco (Ed.), Creativity research handbook. Cresskill, NJ: Hampton Press.

Dow, G. T., \& Mayer, R. E. (2004). Teaching students to solve insight problems: Evidence for domain specificity in creativity training. Creativity Research Journal, 16, 389-402.

Eyrolle, H., \& Cellier, J. M. (2000). The effects of interruptions in work activity: Field and laboratory results. Applied Ergonomics, $31,537-543$.

Fisher, C. D. (1998). Effects of external and internal interruptions on boredom at work: Two studies. Journal of Organizational Behavior, $19,503-522$.

Fleck, J. I., \& Weisberg, R. W. (2004). The use of verbal protocols as data: An analysis of insight in the candle problem. Memory \& Cognition, 32, 990-1006.

Green, P., \& Skinner, D. (2005). Does time management training work? An evaluation. International Journal of Training and Development, 9, 124-138.

Jett, Q. R., \& George, J. M. (2003). Work interrupted: A closer look at the role of interruptions in organizational life. Academy of Management Review, 28, 494-507.

Kirmeyer, S. L. (1988). Coping with competing demands-Interruption and the type-a pattern. Journal of Applied Psychology, 73, 621-629.
König, C. J., Kleinmann, M., \& Höhmann, W. (2004, August). Is a quiet hour an effective time management technique? Paper presented at the Academy of Management, New Orleans, LA.

Macan, T. H. (1994). Time management: Test of a process model. Journal of Applied Psychology, 79, 381-391.

Nandhakumar, J., \& Jones, M. (2001). Accounting for time: Managing time in project-based team working. Accounting, Organizations and Society, 26, 193-214.

Norman, D. A., \& Bobrow, D. G. (1975). Data-limited and resourcelimited processes. Cognitive Psychology, 7, 44-64.

Olton, R. M. (1979). Experimental studies of incubation: Searching for the elusive. Journal of Creative Behavior, 13, 9-22.

Patrick, C. (1938). Scientific thought. Journal of Psychology, 5, 55-83.

Runco, M. A., \& Sakamoto, S. O. (1999). Experimental studies of creativity. In R. J. Sternberg (Ed.), Handbook of creativity (pp. 62-92). Cambridge, UK: Cambridge University Press.

Segal, E. (2004). Incubation in insight problem solving. Creativity Research Journal, 16, 141-148.

Seifert, C. M., Meyer, D. E., Davidson, N., Patalano, A. L., \& Yaniv, I. (1995). Demystification of cognitive insight: Opportunistic assimilation and the prepared-mind perspective. In R. J. Sternberg (Ed.), The nature of insight (pp. 65-124). Cambridge, MA: MIT Press.

Smith, S. M. (1995). Fixation, incubation, and insight in memory and creative thinking. In S. M. Smith, T. B. Ward \& R. A. Finke (Eds.), The creative cognition approach (pp. 135-156). Cambridge, MA: MIT Press.

Smith, S. M., \& Blankenship, S. E. (1991). Incubation and the persistence of fixation in problem-solving. American Journal of Psychology, 104, 61-87.

Sternberg, R. J., \& Davidson, J. E. (1999). Insight. In M. A. Runco \& S. R. Pritzker (Eds.), Encyclopedia of creativity (Vol. 2, pp. 57-69). London: Academic Press.

Wallas, G. (1926). The art of thought. New York: Harcourt.

Weisberg, R. W. (1995). Prolegomena to theories of insight in problem solving: A taxonomy of problems. In R. J. Sternberg \& J. E. Davidson (Eds.), The nature of insight (pp. 157-196). London: MIT Press.

Wells, D. H. (1996). Forced incubation. Creativity Research Journal, 9 , 407-409.

Zijlstra, F. R. H., Roe, R. A., Leonora, A. B., \& Krediet, I. (1999). Temporal factors in mental work: Effects of interrupted activities. Journal of Occupational and Organizational Psychology, 72, 163-185. 